\section{OPEN JOURNAL SYSTEMS \\ ISSN:2237-2202}

Available on line at Directory of Open Access Journals

Journal of Hyperspectral Remote Sensing v.7, n.5 (2017) 299-305

www.periodicos.ufpe.br/revistas/jhrs
Journal of

Hyperspectral

Remote Sensing

\title{
Application of the SEBAL model for determining the net radiation in the Cariri Cearense region
}

\author{
Maria T. B. do Nascimento", João A. S. da Silva ${ }^{* *}$, Carlos W. de Oliveira ${ }^{* * *}$, Ana C. M. Meirele ${ }^{* * * *}$, Francisco L. F. \\ Ferreira $^{* * * * *}$ \\ *Graduando em Agronomia, Bolsista PIBIC, CCAB/UFCA, Crato, Ceará, , \\ ** Mestre em Meteorologia, UFCG, Campina Grande, Paraíba; \\ ${ }_{* * * *}^{*}$ Professor Associado CCAB/UFCA, \\ **** Professora Adjunta CCAB/UFCA, \\ *****Graduando em Agronomia, Bolsista LEMGE, CCAB/UFCA, Crato, Ceará.
}

Received 19 September 2017; accepted 20 October 2017

\begin{abstract}
The urbanization process has contributed directly to the variation of climatic conditions, since this is mainly due to the substitution of vegetation cover for the construction of urban environments. The removal of these areas of vegetation implies changes in their albedo patterns and monochromatic reflectance causing an irregularity in the radiation distribution cycle. Thus, the objective of this work is to determine the balance of radiation in the Cariri Cearense region, by remote sensing using the algorithm SEBAL (Surface Energy Balance Algorithm for Land) and Landsat 5TM images 2616/65 on 07/30/2006, 08/18/2007, 09/21/2008, 09/24/2009, 10/13/2010, 08/29/2011. For a more detailed appreciation of the values of net radiation $(\mathrm{Rn})$, three targets were chosen in the scene, which were: native forest, sparse forest, exposed soil area. All these targets located to windward of the Chapada do Araripe. The Rn estimates showed an increase in dense native forest regions due to the greater vegetative area, in all the images studied. The values for sparse forest and exposed soil were lower.

Keywords: Chapada do Araripe, remote sensing, Landsat.
\end{abstract}

\section{Aplicação do modelo SEBAL para determinação do saldo de radiação na região do Cariri Cearense}

\section{Resumo}

O processo de urbanização tem contribuído diretamente para a variação de condições climáticas, visto que isso se dá principalmente por conta da substituição da cobertura vegetal para a construção de ambientes urbanos. A retirada dessas áreas de vegetação implica mudanças nos seus padrões de albedo e reflectância monocromática fazendo com que ocorra uma irregularidade no ciclo de distribuição de radiação. Assim, o objetivo desse trabalho é determinar o saldo de radiação dentro da região do Cariri Cearense, por de sensoriamento remoto fazendo uso do algoritmo SEBAL (Surface Energy Balance Algorithm for Land) e imagens Landsat 5TM 2616/65 nas datas 30/07/2006, 18/08/2007, 21/09/2008, 24/09/2009, 13/10/2010, 29/08/2011. Para uma apreciação mais detalhada dos valores de saldo de radiação (Rn), foram escolhidos três alvos na cena, os quais foram: floresta nativa, floresta rala, área de solo exposto. Todos estes alvos localizados a barlavento da Chapada do Araripe. As estimativas do Rn mostraram um aumento nas regiões de floresta nativa densa devido a maior área vegetativa, em todas as imagens estudadas. Os valores para floresta rala e solo exposto foram menores.

Palavras-Chave: Chapada do Araripe, sensoriamento remoto, Landsat.

\section{Introdução}

A região do Cariri conta com um elevado índice populacional e, além disso, se destaca por apresentar maior desenvolvimento em relação as outras cidades do Ceará, fato esse, que resulta na maior taxa de urbanização, e consequentemente isso implica na questão da variação da temperatura, visto que é uma região que já apresenta um clima bastante quente. $\mathrm{O}$ processo de urbanização tem contribuído diretamente para a variação de condições climáticas, visto que isso se dá principalmente por conta da substituição da cobertura vegetal para a construção de ambientes urbanos. A substituição da cobertura natural da superfície pela enorme concentração de asfalto e concreto é de forma geral um grande problema, levando em conta que as plantas desempenham função fundamental para partilha de radiação ao contrário dos elementos urbanísticos que absorvem de 98 a 99,9\% da radiação solar que atinge a superfície. (Lombardo, 1985). Diante de tal vertente tem-se por uma importante ferramenta a aplicação de 
algumas técnicas de sensoriamento remoto para determinação do saldo de radiação na região do Cariri. Essas técnicas têm se demonstrado uma alternativa atraente para estimativa dos fluxos de energia e massa no sistema solo-atmosfera em escala regional, com bom nível de exatidão e a baixo custo (Barr et al., 2006). As análises contam com a ajuda de vários modelos de algoritmos que facilitam no levantamento de dados, e um dos mais usados em diversos estudos é o SEBAL (Surface Energy Balance Algorithm for Land), proposto por Bastiaanssen et al. (1998). Para Nicácio (2008), o SEBAL é um algoritmo voltado para o estudo de fluxo de calor e estimativa da evapotranspiração que se destaca por fazer poucas relações e 176 suposições empíricas e por fazer uso de dados multiespectrais de satélites complementados com poucas informações de superfície (como temperatura do ar e velocidade do vento) que são facilmente obtidas nas estações meteorológicas. (Weligepolage, 2005).

Assim, o objetivo desse trabalho é determinar o saldo de radiação dentro da região do Cariri Cearense, através da aplicação de técnicas de sensoriamento remoto fazendo uso do algoritmo SEBAL (Surface Energy Balance Algoritm for Land) e imagens Landsat 5TM 2616/65 nas datas
30/07/2006, 18/08/2007, 21/09/2008, 24/09/2009, 13/10/2010, 29/08/2011. Para a referida determinação tomou-se, mais especificamente, o recorte das cidades de Juazeiro do Norte, Crato e Barbalha, que se destacam por apresentar índices de população mais elevados.

\section{Material e métodos}

A região do cariri (Figura 1) está localizada no sul do Ceará e possui uma área total de 4.115,82 $\mathrm{km}^{2}$. Conta com uma população estimada em 1.386.310 habitantes, possuindo uma densidade demográfica de 48,4 hab. $\mathrm{km}^{-2}$. Tem como clima predominante o semiárido, caracterizado por ter seu período chuvoso restrito a 3 ou 4 meses do ano e alta biodiversidade. Dentro dos municípios de Crato e Barbalha existe uma unidade de conservação administrada pelo Instituto Chico Mendes, conhecida como Floresta Nacional do Araripe, ou FLONA Araripe. O clima da FLONA-ARARIPE é do tipo Aw', característico de "Clima Tropical Chuvoso" (classificação de Köppen), com precipitação média anual de $1.033 \mathrm{~mm}$, distribuída numa estação chuvosa que vai de janeiro a maio (DNPM, 1996).

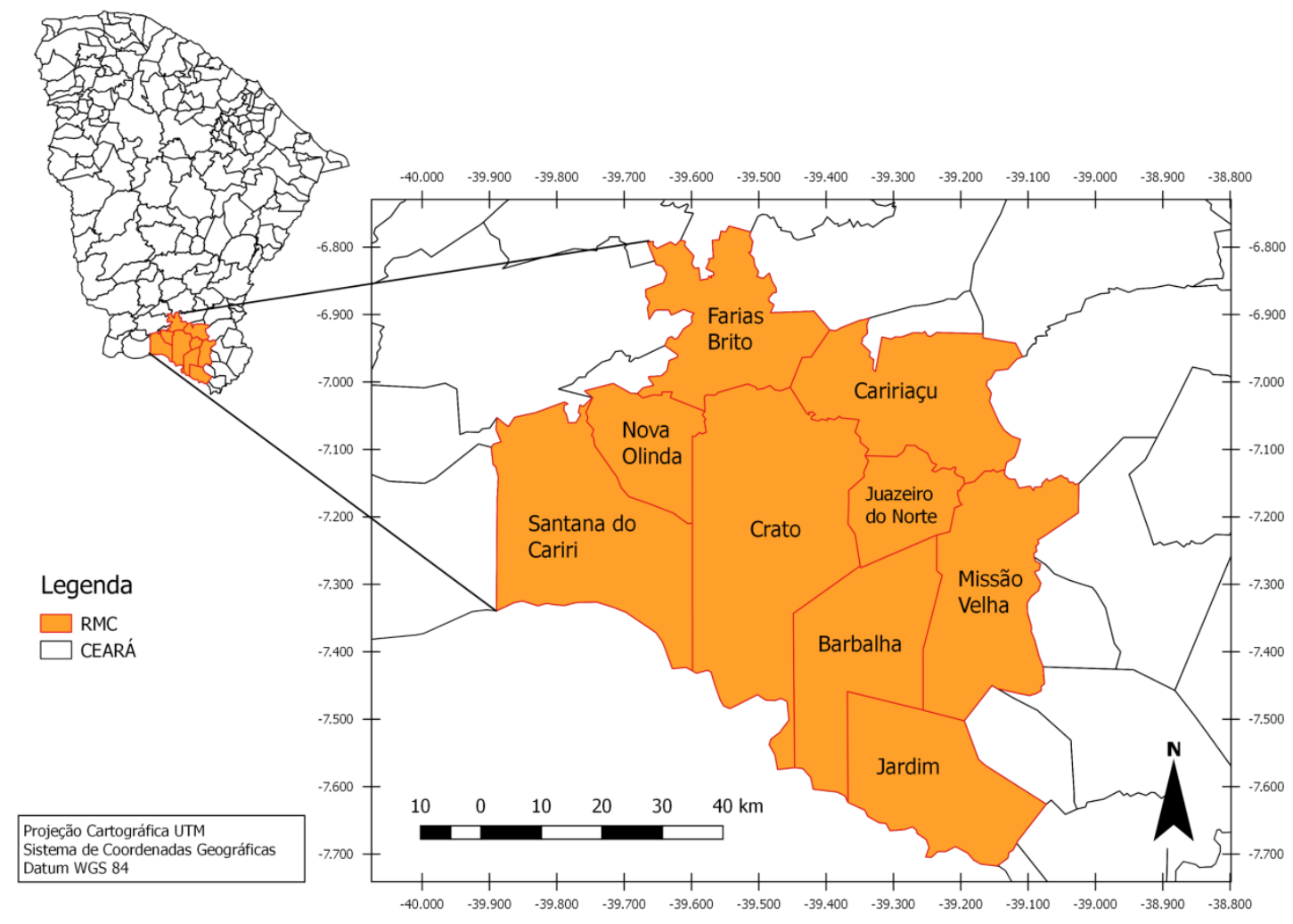

Figura 1 - Mapa da região Metropolitana do Cariri. 
A determinação do Balanço de Radiação foi feita a partir de imagens do sensor TM (Thematic Mapper), do satélite Landsat 5, adquiridas no site do Instituto Nacional de Pesquisas Espaciais - INPE (www.inpe.br) compostas por 7 bandas espectrais.

A imagem utilizada foi da órbita 217 ponto 65, nas datas de 30/07/2006, 18/08/2007, 21/09/2008, 24/09/2009, 13/10/2010, 29/08/2011. O processamento da imagem de satélite foi feito com o auxílio de modelos desenvolvidos através da ferramenta Model Maker do software Erdas Imagine 8.5.

Para determinação do Saldo de Radiação na Região Metropolitana do Cariri foram desenvolvidos modelos baseados nos algoritmos SEBAL (Surface Energy Algorithm for Land) conforme as etapas computacionais apresentada no fluxograma da Figura 2.

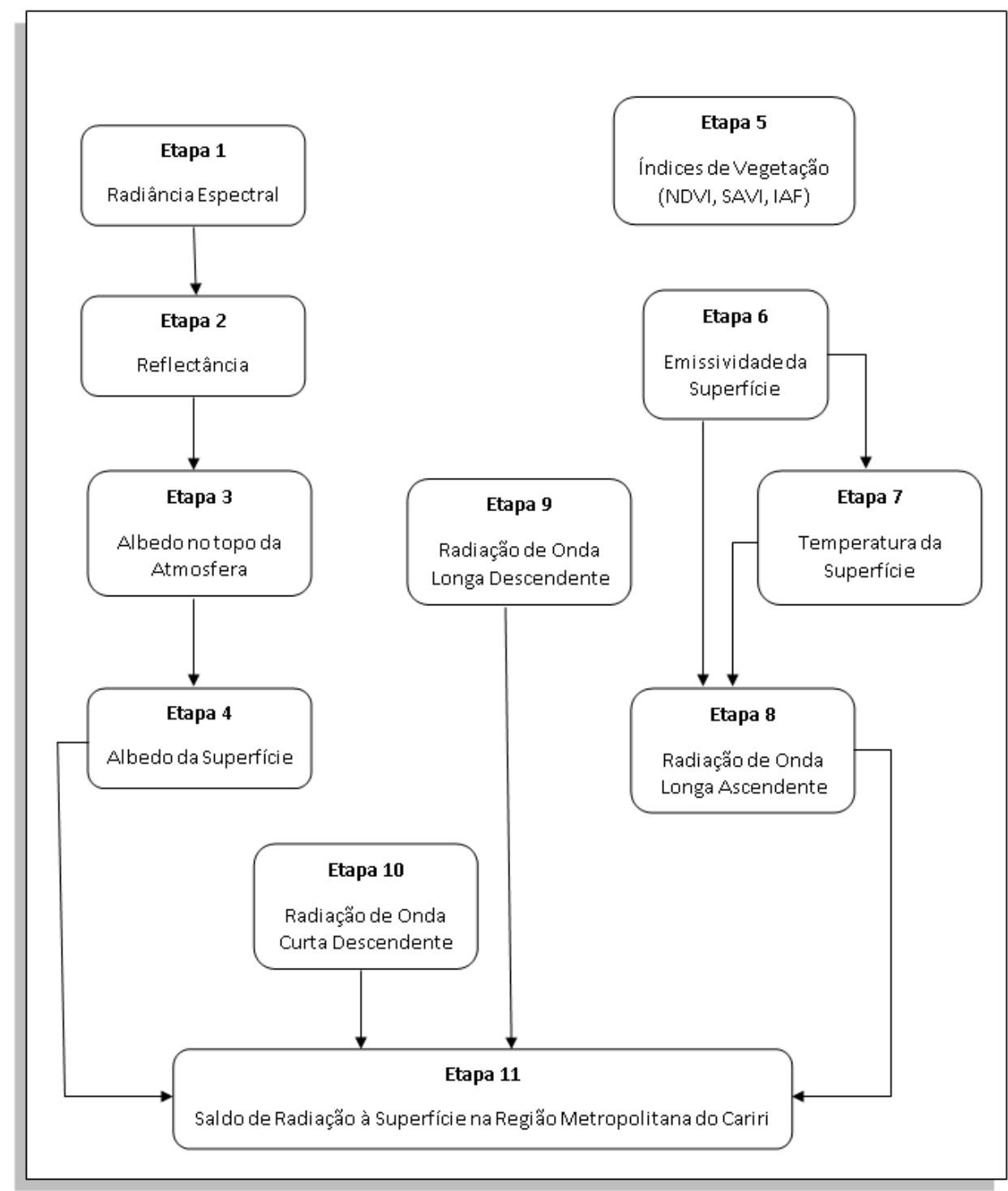

Figura 2 - Fluxograma das etapas computacionais do processamento do Balanço de Radiação à Superfície na Região Metropolitana do Cariri.

A primeira etapa do algoritimo é calcular a calibração radiométrica é a conversão do numero digital (DN) ou intensidade de cada pixel da imagem em radiância espectral ou calibração radiométrica e utiliza-se a equação abaixo para conversão. Utilizouse metodologia e dados de constantes de calibração espectral do sensor, segundo Chander e Markham (2003), apresentados na Tabela 1. 
Tabela 1. Descrição das bandas do Mapeador Temático (TM) do satélite Landsat 5 com os correspondentes intervalos de comprimento de onda, coeficientes de calibração (radiância mínima - LMIN e máxima - LMAX) e irradiâncias espectrais no topo da atmosfera (TOA).

\begin{tabular}{|c|c|c|c|c|c|}
\hline \multirow[t]{2}{*}{ Bandas } & \multirow[t]{2}{*}{$\begin{array}{c}\text { Comprimento de } \\
\text { Onda } \\
(\mu \mathrm{m})\end{array}$} & \multicolumn{2}{|c|}{$\begin{array}{c}\text { Coeficiente de } \\
\text { Calibração } \\
\left(\mathrm{Wm}^{-2} \mathbf{s r}^{-1} \mu^{-1}\right) \\
\text { Após } 05 / 05 / 2003 \\
\end{array}$} & \multirow[t]{2}{*}{$\begin{array}{c}\text { Irradiância Espectral } \\
\text { no Topo da Atmosfera } \\
\text { ESUN } \\
\left(\mathbf{W m}^{-2} \mu^{-1}\right)\end{array}$} & \multirow[t]{2}{*}{$\begin{array}{c}\text { Coeficientes cômputo do } \\
\text { albedo topo da atmosfera } \\
\text { W }\end{array}$} \\
\hline & & $\mathbf{L}_{\text {MIN }}$ & $\mathbf{L}_{\mathbf{M A X}}$ & & \\
\hline $\begin{array}{l}\text { Banda } 1 \\
\text { (azul) }\end{array}$ & $0,45-0,52$ & $-1,52$ & 193,00 & 1957 & 0,293 \\
\hline $\begin{array}{l}\text { Banda } 2 \\
\text { (verde) }\end{array}$ & $0,52-0,60$ & $-2,84$ & 365,00 & 1826 & 0,274 \\
\hline $\begin{array}{l}\text { Banda } 3 \\
\text { (vermelho) }\end{array}$ & $0,63-0,69$ & $-1,17$ & 264,00 & 1554 & 0,233 \\
\hline $\begin{array}{l}\text { Banda } 4 \\
\text { (IV- } \\
\text { próximo) }\end{array}$ & $0,76-0,90$ & $-1,51$ & 221,00 & 1036 & 0,157 \\
\hline $\begin{array}{l}\text { Banda } 5 \\
\text { (IV-médio) }\end{array}$ & $1,55-1,75$ & $-0,37$ & 30,20 & 215,0 & 0,033 \\
\hline $\begin{array}{l}\text { Banda } 6 \\
\text { (IV-termal) }\end{array}$ & $10,42-12,50$ & 1,24 & 15,303 & - & - \\
\hline $\begin{array}{l}\text { Banda } 7 \\
\text { (IV-médio) }\end{array}$ & $2,08-2,35$ & $-0,15$ & 16,50 & 80,67 & 0,011 \\
\hline
\end{tabular}

Uma vez feita às calibrações outra principal etapa é o computo das variáveis biofísicas tais como NDVI. O calculo é feito pela razão da diferença do infravermelho-próximo e vermelho e a diferença entre eles. Equação 1.

NDVI $=\left(\rho \_I V-\rho \_v /\left(\rho \_I V+\rho \_v\right)(1)\right.$

( $\left.\rho_{\text {_IV }}\right)$ e $\left(\rho_{-} v\right)$, corresponde, respectivamente, a reflectância das bandas 4 e 3 do landsat $5-$ TM. O NDVI, usado para identificar a presença, quantidade e condição da vegetação verde na superfície varia entre os valores de -1 a 1 ; onde valores positivos correspondem há áreas vegetadas ou com algum tipo de vegetação que dependem de acordo com a atividade fotossintética das plantas nessa área e sua densidade, enquanto locais com água e com nuvens geralmente ficam negativos.

Para o cômputo do albedo da superfície é necessário valor do albedo planetário ou albedo do topo da superfície, que é calculado através de uma combinação linear das reflectância espectrais $\left(\rho_{\lambda}\right)$, calculados anteriormente, e o coeficiente de regressão $\left(w_{\lambda}\right)$, de acordo com a equação:

$\alpha_{\text {toa }}=\left(w_{1} \cdot \rho_{1}\right)+\left(w_{2} \cdot \rho_{2}\right)+\left(w_{3} \cdot \rho_{3}\right)+\left(w_{4} \cdot \rho_{4}\right)+\left(w_{5} \cdot \rho_{5}\right)+$ (radiação de onda longa emitida pela atmosfera; $R_{L \uparrow}$ a

(2)

Os coeficientes de regressão linear da equação anterior $\left(w_{1}, w_{2}, \ldots w_{7}\right)$, são calculados pela equação:

$w_{\lambda}=E S U N_{\lambda} / \Sigma E S U N_{\lambda}$

Para o Landsat 5-TM, os valores de $\mathrm{w}_{\lambda}$, são apresentados na tabela 1 .

Depois de computados todos os processos anteriores calcula-se o Albedo da superfície ou albedo corrigido, que é um primeiro termo do balanço de radiação que é computado através da equação seguinte:

$\alpha=\left(\alpha_{\text {toa }}-\alpha_{\text {path_radiance }}\right) / T_{s w}^{2}$

O saldo de radiação à superfície é obtido utilizando a equação de balanço de radiação à superfície (Silva et al., 2005).

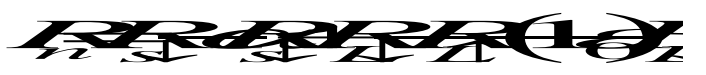

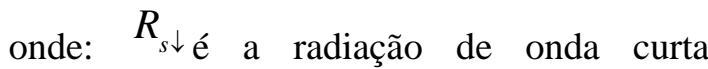
incidente; $\alpha$ o albedo corrigido do pixel; $R_{L \downarrow}$ a radiação de onda longa emitida pelo pixel; ${ }^{\varepsilon_{O}}$ a emissividade do pixel.

Simplificando a expressão anterior, obtemos: 


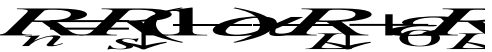

\section{Resultados e discussão}

A cena estudada constitui uma área bem heterogênea formada pela Região metropolitana do Cariri que engloba os municípios de Barbalha Juazeiro do Norte e Crato seus centros urbanos e em seu redor áreas rurais de pastagem, agricultura de subsistência e vegetação nativa densa (Floresta Nacional do Araripe). Conforme apresentada na Figura 3, que é uma composição RGB formada pelas bandas 4, 3 e 2 do sensor TM.

A região localiza-se a barlavento da Chapada do Araripe apresenta uma vegetação que se mantém verde por quase todo o ano. Já a vegetação da Florestal Nacional do Araripe, que se localiza ao centro da Figura 2 em tonalidade vermelha, é uma vegetação de grande porte e bem adensada com boa disponibilidade hídrica. É possível também identificar áreas com solo exposto, com tonalidade esbranquiçada além da variação da densidade da vegetação nativa, indo de uma tonalidade verde escuro (mais densa), a um tom esverdeado claro (menos densa).

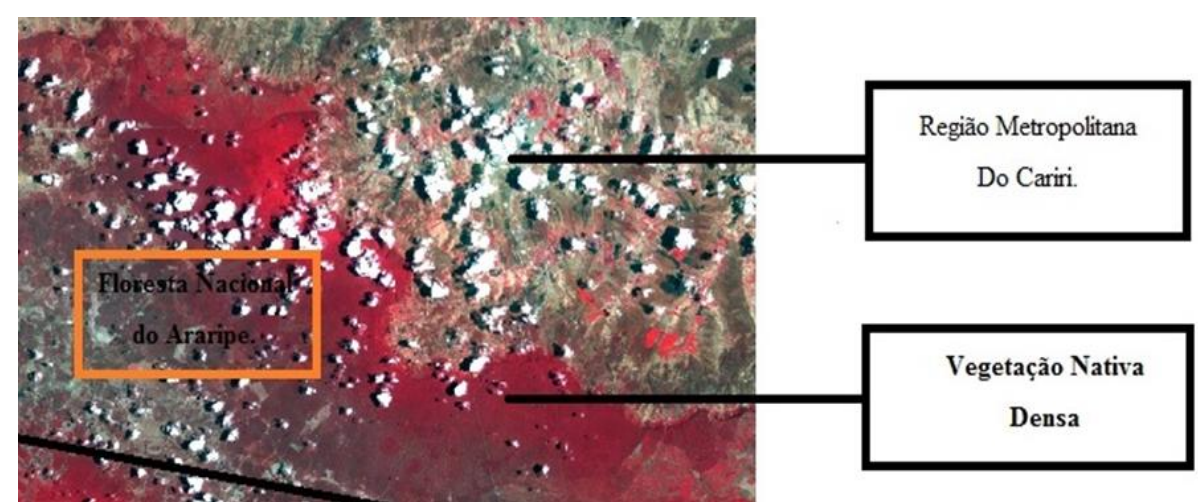

Figura 3 - Cena estudada em composição RGB para as bandas 4, 3 e 2 Landsat 5-TM, dia 29/08/2011.

Neste estudo, os componentes do Balanço Energético foram estimados em valores instantâneos para o momento da passagem do satélite. Para uma apreciação mais detalhada dos valores de saldo de radiação (Rn), NDVI foram escolhidos três alvos na cena. Os quais foram: floresta nativa, floresta rala, área de solo exposto. Todos estes alvos localizados a barlavento da Chapada do Araripe.

A cena estudada teve valores de NDVI médio igual a 0,63 apresentando índice de área foliar (IAF) entre 1,20 e 1,45 para áreas de floresta nativa rala, no entanto, apresentaram um NDVI em torno de $0,54 \mathrm{e}$ IAF entre 0,87 e 1,02. Já as áreas de centros urbanos apresentaram um NDVI aproximadamente de 0,12 com IAF entre 0,01 e 0,02 .

Bezerra et al. (2011) encontrou na mesma região para segundo período do ano (seco), em locais de mata nativa Caatinga NDVI em torno de 0,20 , já para locais de pediplano com algum grau de antropização valores de NDVI de 05, no topo da chapada do Araripe em locais onde a floresta se apresenta densa valores de 0,84. Estes valores corroboram com Huete \& Tucker (1991), os valores de NDVI para solo expostos, geralmente, ficam entre 0,05 a 0,30 e, devido à grande variabilidade das propriedades ópticas do solo, citam os autores, que não é possível definir um intervalo de valores rigorosos de NDVI para os solos com pouca ou nenhuma vegetação.

Nas Tabelas 2, 3, 4, 5, 6 e 7, são apresentados os resumos estatísticos das áreas selecionadas para análise mais detalhada do comportamento do saldo de radiação $(\mathrm{Rn})$.

Tabela 2 - Resumo estatístico dos resultados do saldo de radiação das áreas, imagem 216/65 de 29/08/2011.

\begin{tabular}{lccc}
\hline \multicolumn{1}{c}{ Área } & Média $\left(\mathbf{w m}^{-\mathbf{2}}\right)$ & Mediana $\left(\mathbf{w m}^{-\mathbf{2}}\right)$ & Desvio Padrão $\left(\mathbf{w m}^{-2}\right)$ \\
\hline Floresta densa & 635,85 & 636,08 & 5,60 \\
\hline Floresta rala & 595,95 & 600,80 & 26,35 \\
\hline Solo nu & 493,34 & 494,56 & 38,27 \\
\hline
\end{tabular}


Tabela 3 - Resumo estatístico dos resultados do saldo de radiação das áreas, imagem 216/65 de 13/10/2010.

\begin{tabular}{lccc}
\hline \multicolumn{1}{c}{ Área } & Média $\left(\mathbf{w m}^{-2}\right)$ & Mediana $\left(\mathbf{w m}^{-2}\right)$ & Desvio Padrão $\left(\mathbf{w m}^{-2}\right)$ \\
\hline Floresta densa & 675,1 & 674,72 & 18,86 \\
\hline Floresta rala & 592,71 & 592,9 & 29,7 \\
\hline Solo nu & 472,64 & 463,05 & 30,89 \\
\hline
\end{tabular}

Tabela 4 - Resumo estatístico dos resultados do saldo de radiação das áreas, imagem 216/65 de 24/09/2009.

\begin{tabular}{lccc}
\hline \multicolumn{1}{c}{ Área } & Média $\left(\mathbf{w m}^{-\mathbf{2}}\right)$ & Mediana $\left(\mathbf{w m}^{-2}\right)$ & Desvio Padrão $\left(\mathbf{w m}^{-\mathbf{2}}\right)$ \\
\hline Floresta densa & 703,45 & 703,51 & 5,29 \\
\hline Floresta rala & 608,92 & 615,94 & 33,03 \\
\hline Solo nu & 471,79 & 466,88 & 39,62 \\
\hline
\end{tabular}

Tabela 5 - Resumo estatístico dos resultados do saldo de radiação das áreas, imagem 216/65 de 21/09/2008.

\begin{tabular}{lccc}
\hline \multicolumn{1}{c}{ Área } & Média $\left(\mathbf{w m}^{-\mathbf{2}}\right)$ & Mediana $\left(\mathbf{w m}^{-2}\right)$ & Desvio Padrão $\left(\mathbf{w m}^{-\mathbf{2}}\right)$ \\
\hline Floresta densa & 704,96 & 704,96 & 5,36 \\
\hline Floresta rala & 617,91 & 621,76 & 28,25 \\
\hline Solo nu & 493,24 & 483,8 & 39,76 \\
\hline
\end{tabular}

Tabela 6 - Resumo estatístico dos resultados do saldo de radiação das áreas, imagem 216/65 de 18/08/2007.

\begin{tabular}{lccc}
\hline \multicolumn{1}{c}{ Área } & Média $\left(\mathbf{w m}^{-2}\right)$ & Mediana $\left(\mathbf{w m}^{-2}\right)$ & Desvio Padrão $\left(\mathbf{w m}^{-2}\right)$ \\
\hline Floresta densa & 803,79 & 803,94 & 4,84 \\
\hline Floresta rala & 725,2 & 728,72 & 23,5 \\
\hline Solo nu & 635,62 & 629 & 33,36 \\
\hline
\end{tabular}

Tabela 7 - Resumo estatístico dos resultados do saldo de radiação das áreas, imagem 216/65 de 30/07/2006.

\begin{tabular}{lccc}
\hline \multicolumn{1}{c}{ Área } & Média $\left(\mathbf{w m}^{-2}\right)$ & Mediana $\left(\mathbf{w m}^{-2}\right)$ & Desvio Padrão $\left(\mathbf{w m}^{-2}\right)$ \\
\hline Floresta densa & 712,24 & 712,29 & 4,51 \\
\hline Floresta rala & 672,66 & 674,99 & 16,51 \\
\hline Solo nu & 575,19 & 574,05 & 34,39 \\
\hline
\end{tabular}

Silva et al., (2011) em medições a campo de pomares de Cana de Açucar irrigados, no semiárido brasileiro, encontrou valores baixos do saldo de radiação de $174 \mathrm{~W} \mathrm{~m}^{-2}$. Esses baixos valores estavam associados além da baixa incidência de radiação no período de observação, aos menores valores de IAF, causados pelo corte da cana, e consequente emissão de energia para atmosfera negativando o saldo de ondas longas. Os valores de floresta densa mostraram-se bem elevados indo de 635 a $803 \mathrm{Wm}^{-2}$ 
tanto devido ao período medido quando as chuvas sessam na região e a atmosfera apresenta maior transmissividade.

Os valores médios obtidos para floresta rala de 595,95 a $735 \mathrm{~W} \mathrm{~m}^{-2}$, são superiores a valores encontrados para áreas de Caatinga. Silans \& Silva (2007), estudando o fluxo de calor sensível e evapotranspiração na caatinga em São João do Cariri, na Paraíba, obtiveram valores de Radiação Líquida variando de 400 a $500 \mathrm{~W} \mathrm{~m}^{-2}$ em períodos próximos ao meio-dia.

\section{Conclusões}

As estimativas do Saldo de Radiação (Rn), mostraram um aumento nas regiões de floresta nativa densa, fato decorrente de uma maior área de vegetação, em todas as imagens estudadas. Os valores para floresta rala e solo exposto foram menores. O IAF obtido nas áreas de floresta rala os valores oscilaram entre 1,20 e 1,45, porém nas áreas representativas de solo nu foi obtido 0,01 e 0,02 , devido a falta de vegetação, visto que com o solo exposto a radiação não é absorvida e é refletida. $\mathrm{O}$ modelo SEBAL se mostrou muito eficiente no que se refere aos valores obtidos de $\mathrm{Rn}$.

\section{Agradecimentos}

Ao CNPq pelo programa de bolsas
PIBIC/UFCA/CNPq.

\section{Referências}

Barr, A.G., Morgenstern, K., Black, T.A., Mcgaughey, J.H., Nesic, Z., 2006. Surface energy balance closure by the eddy-covariance method above three boreal forest stands and implications for the measurement of the $\mathrm{CO} 2$ flux. Agricultural and Forest Meteorology 140, 322-337.

Bastiaanssen, W.G., Menenti, M., Fedds, R.A.,
Holtslag, A.A., 1998 A remote sensing surface energy balance algorithm for land (SEBAL) 1. Formulation. Journal of hidrology 212-213, 198212.

Bezerra, M.V.C., Silva, B.B.da, Bezerra, B.G., 2011. Avaliação dos efeitos atmosféricos no albedo e NDVI obtidos com imagens de satélite. Revista Brasileira de Engenharia Agrícola e Ambiental 15, 709-717.

Conçeição, A.F., Furtado, A.L.dosS., Filardi, A.L., Ninomya, M., Lourenço, F.R., 2008. Remote Sensing and GIS applications as tool for planning and control FMD in Brazilian Border, Campinas.

Huete, A.R., Tucker, C.J., 1991. Investigation of soil influence in AVHRR red and near infrared vegetation index imagery. International Journal of Remote Sensing 12, 1223-1242.

Lombardo, M.A., 1985. Ilhas de Calor nas Metrópoles: o exemplo de São Paulo. HUCITEC, São Paulo.

Nicácio, R.M., 2008, Evapotranspiração real e umidade do solo usando dados de sensores orbitais e a metodologia SEBAL na bacia do rio São Francisco. Tese (Doutorado). Rio de Janeiro, UFRJ.

Silva, B.B.da, Lopes, G.M., Azevedo, P.V., 2005. Balanço de radiação em áreas irrigadas utilizando imagens Landsat 5 - TM. Revista Brasileira de Meteorologia 20, 243-252.

Silva, T.G.F.. Moura, M.S.B, Zolnier, S., Soares, J.M., de Souza, L.S.B., Brandão, E.O., 2011. Variação do balanço de radiação e de energia da cana-de-açúcar irrigada no semiárido brasileiro. Revista Brasileira de Engenharia Agrícola e Ambiental 15, 139-147.

Weligepolage, K., 2005. Estimation of spatial and temporal distribuition of evapotranspirationby satellite remote sensing - A case study in Hupselse Beek. Thesis (Master). International Institute for Geo- information Science and Earth Observation. 